\title{
FREQUENCY-HOPPING TRELLIS-CODED 8-DPSK FOR INDOOR COMMUNICATIONS
}

\author{
R. AGUSTI and G. FEMENIAS
}

Universitat Politècnica de Catalunya (UPC)

Apdo. 30.002, 08080 Barcelona (Spain)

\begin{abstract}
In this paper we propose a TDMA Slow Frequency Hopping-Trellis Coded 8DPSK scheme for indoor mobile radio communications. In order to assess the performance of this scheme a GWSSUS (Gaussian Wide Sense Stationary Uncorrelated Scattering) continuous channel model with an exponential Power Delay Profile has been adopted. This channel model allows us to analize transmission rates much lower than the inverse of the r.m.s. delay spread. Bit error rate (BER) is obtained, through Monte Carlo computer simulation, as a function of signal to noise ratio (SNR) and with normalized frequency-hopping separation and number of hopping-frequency channels as parameters.
\end{abstract}

\section{INTRODUCTION}

The performance of an indoor mobile radio system operating in the UHF band is severely affected by the multipath propagation environment. The presence of deep fades, characteristic of the Rayleigh fading channel, produce high error rates even with high average signal to noise ratios. To counteract the effects of the fading one can resort to coding, diversity or combined coding/diversity schemes.

Conventional block and convolutional coding schemes have proved to be very powerful in counteracting the fading effects when used in conjunction with interleaving strategies. Unfortunately, the high delay associated with the interleaving process when applied to quasi-static indoor mobile radio channels (hundred of ms. due to the fact that the maximum doppler frequency involved is lower than some $\mathrm{Hz}$.) makes such coding techniques inappropriate. Moreover, the extra bandwidth required causes an additional cost to be paid in terms of spectrum efficiency. Trellis Coded Modulation (TCM), as introduced by Ungerboeck [1] [2], has recently become a popular means of achieving coding gain on the AWGN channel and also on the Rayleigh fading channel, without extra bandwidth requirements and with simple decoders utilizing the Viterbi algorithm. Thus, the lost of spectrum efficiency due to coding can be overcome using TCM schemes. However, the interleaving associated delay will still remain [3].

Diversity techniques have often been also invoked to combat the Rayleigh fading and the obtained results, usually with space diversity reception, have actually shown its effectiveness. Yet, the requirements for a more complex receiver have avoided their widespread adoption.

The more recent standard systems of mobile communications such as GSM, DEC, as well as other systems presently envisaged, use in some extent combined coding/diversity schemes by means of Frequency Hopping (FH) techniques in order to take advantage of the intrinsic frequency diversity that the FH enable. In this paper we propose a TDMA combined Slow FH-TCM 8DPSK scheme for indoor mobile communications that avoids the above mentioned delay and extra bandwidth requirements without renouncing the beneficial effects of using coding techniques. Moreover, with the proposed technique, the intrinsic diversity atributes that the FH schemes present are exploited without significantly increasing the receiver complexity. In short, the proposed scheme uses the FH strategy to make the channel memoryless. In this case, the delay of interleaving would no longer be controlled by the coherence time of the channel but by the FH period code and the TDMA system efficiency.

\section{SYSTEM MODEL}

The equivalent baseband transmission system is shown in Fig. 1. A non-coherent modulation has been adopted. We have assumed the overall transfer function to be, in absence of channel distortion, a raised-cosine with a 0.5 roll-off parameter equally split into transmitter and receiver. In order to emphasize only the effects of the coding/diversity set-up, perfect automatic frequency control (AFC) and clock recovery are assumed.

At the emitter, a data input bit pair $s_{i}=\left(s_{0, j}, s_{1, j}\right)$ is fed into a rate $2 / 3$ trellis encoder at time $t=i T$, which generates the coded 3-bit word $m_{i}=\left(m_{0, i}, m_{1, j}, m_{2, j}\right)$ as a function of its state at the instant $t=(i-1) T$ and of the data input symbol $s_{i}$. The octal mapper, applying mapping by set partitioning [1] [2], converts the 3-bit word $m_{i}$ into the coded 8.PSK symbol $a_{i}$ given by

$$
a_{1}=\exp \left\{j \cdot\left(m_{2 j} \cdot \pi+m_{1,} \cdot \frac{\pi}{2}+m_{0, j} \cdot \frac{\pi}{4}\right)\right\}
$$

These coded symbols are fed into the block interleaver that can be regarded as a buffer, with NL rows and NC columns, that 


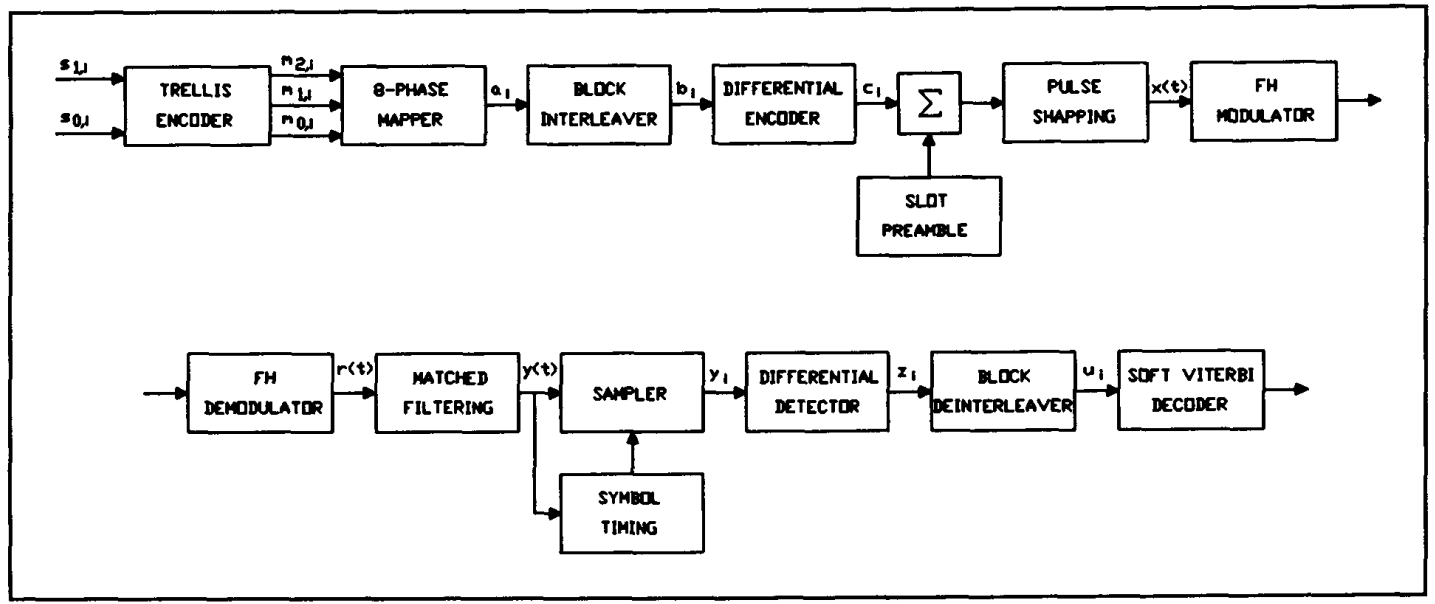

Figure 1. Bit error rate of the FH-TCM-8DPSK system versus signal to noise ratio with normalized hopping frequency separation as parameter.

breaks the channel memory and makes full use of the error correction properties of the Ungerboeck code. The data symbols $\left\{a_{i}\right\}$ are stored into the buffer in successive columns and read out in rows. Differential encoding of the interleaver output symbol, $b_{i}$, yields the coded 8-DPSK symbol

$$
c_{i}=c_{i-1} \cdot b_{i}
$$

The TDMA time slot structure is composed by a preamble, a sync word, a row of 8-DPSK symbols and a spare time. Preamble would allow to stablish the AGC (Automatic Gain Control) and to carry out the clock recovery, and the sync word is devoted to perform the address detection and to indicate the beginning of the data symbols. Once filtered by the transmission filter, $h_{\mathrm{T}}(\mathrm{t})$, these symbols provide the complex baseband transmitted signal,

$$
x(t)=\sum_{k} c_{k} \cdot h_{T}(t-k T)
$$

Finally, to make use of the FH intrinsic diversity, the NL formed slots are modulated according to $\mathrm{NL}$ assigned hopping frequencies. By setting the frequency-hopping separation sufficiently large, a null correlation among the different frequency channels can be assured. Moreover, to guarantee a memoryless channel, at first, NL should be chosen such that its equivalent time span should be equal to or larger than the decoding delay introduced by the soft Viterbi decoder. We will come back on this point later. The number of columns, NC, depends on the frame efficiency of the TDMA scheme. In our analysis this will not be a relevant parameter. In fact, frame duration is considered to be far shorter than the coherence time of the channel, and thus, in order to determine the system performance, a continuous transmission scheme can be assumed.

Assuming, in general, that we are receiving a signal transmitted onto the $j$-th hopping frequency $(j=1,2, \ldots, N L)$, the complex baseband signal at the output of the FH demodulator can be expressed as

$$
r(t)=\sum_{k} c_{k} \cdot H_{c}\left(t ; f_{j}\right) \cdot h_{T}(t-k T)+w_{j}(t)
$$

where $w_{j}(t)$ is an additive complex white Gaussian noise and $\mathrm{H}_{\mathrm{C}}\left(\mathrm{t} ; \mathrm{f}_{\mathrm{j}}\right)$ is a complex-valued Gaussian stochastic process which describes multiplicative non-frequency selective short-term fading, characteristic of the $j$-th radio channel. The received signal is filtered by the reception filter giving,

$$
y(t)=\sum_{k} c_{k} \cdot H_{c}\left(t ; f_{j}\right) \cdot h(t-k T)+n_{j}(t)
$$

where $h(t)$ is the overall impulse response of the transmission channel in absence of Rayleigh distortion and $n_{j}(t)$ is an additive complex Gaussian noise. This signal is sampled by an A/D converter at time $\mathrm{t}_{\mathrm{i}}=\mathrm{i} \mathrm{T}+\tau$, where $-\mathrm{T} / 2 \leq \tau \leq \mathrm{T} / 2$ determines the sampling time, providing the complex sample,

$$
\begin{gathered}
y_{i}=c_{i} \cdot H_{c}\left(t_{i} ; f_{j}\right) \cdot h(\tau)+ \\
+\sum_{k=i} c_{k} \cdot H_{C}\left(t_{i} ; f_{j}\right) \cdot h\left(t_{i}-k T\right)+n_{j}\left(t_{i}\right) .
\end{gathered}
$$

This expression shows that the wanted sample is affected by a term of intersymbol interference and a term of Gaussian noise. 


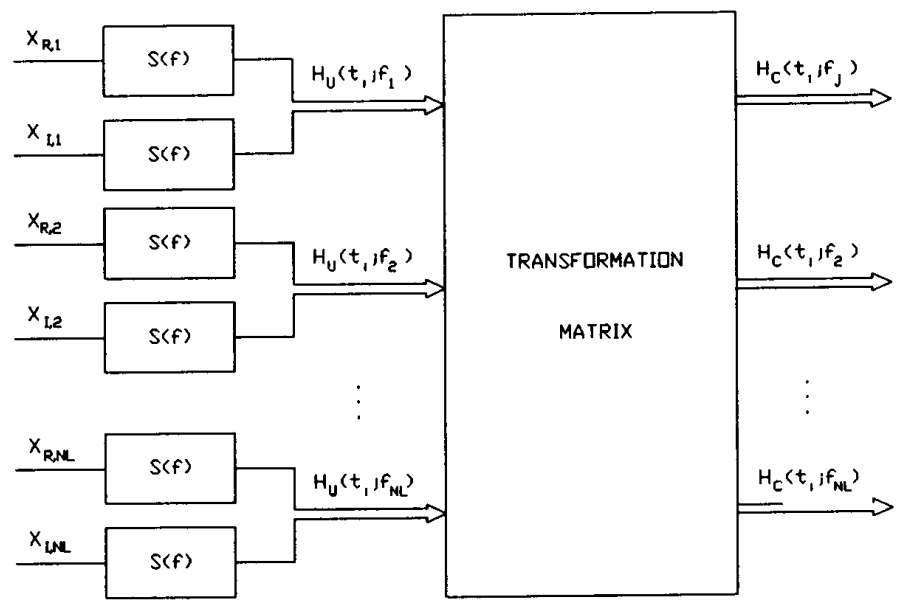

UNCORRELATED

REAL WHITE

GAUSSIAN PROCESSES
TIME CORRELATED

COMPLEX-VALUED

GAUSSIAN PRDCESSES
FREQUENCY AND TIME CORRELATED COMPLEX-VALUED GAUSSIAN PROCESSES

Figure 2. BER of the FH-TCM-8DPSK system versus signal to noise ratio with the number of frequency channels (assumed to be uncorrelated) as parameter.

Assuming a perfect clock recovery, $\tau=0$, we have $h(0)=1$ and $h((i-k) T)=0$, for all $k \neq i$, and so the ISI term vanishes,

$$
y_{i}=c_{i} \cdot H_{c}\left(t_{i} ; f_{j}\right)+n_{j}\left(t_{i}\right)=c_{i} \cdot H_{c, t, J}+n_{j, l}
$$

For the Nyquist system one can assume the noise samples $n_{j, i}$ to be uncorrelated and so, the $\mathrm{j}$-th frequency channel average bit energy to spectral noise power density ratio is, for $\tau=0$,

$$
\frac{E_{b J}}{N_{0}}=\frac{E\left\{\left|H_{C, t, j}\right|^{2}\right\} \cdot E\left\{\left|c_{l}\right|^{2}\right\}}{4 \cdot \sigma^{2}}
$$

where $E[\cdot\}$ denotes expectation and $\sigma^{2}$ is the variance of the thermal noise in each dimension.

Once performed the differential detection, the obtained samples (soft quantized symbols), $z_{\mathrm{i}}$, are stored into the block deinterleaver in successive rows and read out in columns for soft Viterbi decoding. The trellis encoder used at the transmitter side functions as a "finite state machine", then the Viterbi decoder will be optimum for estimating the maximum likelihood coded symbol sequence. But the noise samples at the Viterbi decoder input are non-Gaussian and correlated, for this reason, the use of a proper metric is needed. The cumulative metric that we have used for the path ending at state $\mu$ at time iT is given by the negative increase of the quadratic Euclidian distance over a symbol period [4],

$$
Q_{\mu, i}=-\sum_{k=0}^{L}\left|u_{i-k}-a_{k, i-k}\right|^{2}
$$

where $\mathrm{L}$ represents the delay introduced by the soft Viterbi decoder, and $u_{i}$ is the deinterleaver matrix output symbol at the instant iT.

\section{CHANNEL SIMULATION}

In order to assess the performance of the system shown in Fig.1, a GWSSUS (Gaussian Wide Sense Stationary Uncorrelated Scattering) continuous channel model with an exponential Power Delay Profile, with r.m.s. delay spread given by Ds, has been adopted. This channel model allows us to analize transmission rates much lower than 1/Ds (flat fading).

Figure 2 shows the GWSSUS channel simulation procedure. The $\mathrm{NL}$ signals $\mathrm{H}_{\mathrm{U}}\left(\mathrm{t} ; \mathrm{f}_{\mathrm{j}}\right) \quad(\mathrm{j}=1, \ldots, \mathrm{NL})$ represent independent (uncorrelated) complex-valued Gaussian stochastic processes which describe multiplicative non-frequency selective fading. The envelope $\left|\mathrm{H}_{\mathrm{U}}\left(\mathrm{t} ; \mathrm{f}_{\mathrm{j}}\right)\right|$ and the phase $\operatorname{Arg}\left\{\mathrm{H}_{\mathrm{U}}\left(\mathrm{t} ; \mathrm{f}_{\mathrm{j}}\right)\right\}$ have Rayleigh and $[0,2 \pi]$-Uniform distributions, respectively. Even knowing that, actually, a precise formulation of the doppler power spectrum is not necessary because of the quasi-static nature of the channel makes that all the symbols 
contained in the interleaving matrix "see" practically the same mobile channel, the channel dynamics are useful in the BER calculation procedure in the sense that the inherent averaging is naturally carried out in the computer simulation adopted. Thus, the baseband doppler power spectrum associated with each channel has been taken to be [5],

$$
S(f)=\left\{\begin{array}{cc}
{\left[1-\left(\frac{f}{f_{d}}\right)^{2}\right]^{-1 / 2}} & , \text { for }|f| \leq f_{d} \\
0 & , \text { for }|f|>f_{d}
\end{array}\right.
$$

where $f_{d}$ is the maximum doppler shift frequency. Real and imaginary parts of each channel samples having this spectrum have been generated by shaping two statistically independent white Gaussian random sequences with a 6th order low pass filter.

In fact, we are interested in having frequency channels, characterized as complex-valued stochastic processes, with a correlation function versus the hopping-frequency separation, $\mathrm{C}\left(\mathrm{S}_{\mathrm{f}}\right)$, given by the Fourier transform of the power delay profile

$$
\Phi_{c}(\tau)=\frac{1}{D s} \exp \left(-\frac{\tau}{D s}\right) u(\tau) .
$$

That is,

$$
\begin{gathered}
C\left(S_{f}\right)=E\left[H_{c}^{*}\left(t ; f_{i}\right) \cdot H_{C}\left(t ; f_{i}+S_{f}\right)\right]= \\
=\frac{1}{1+j 2 \pi S_{f} D_{s}} .
\end{gathered}
$$

For this purpose the transformation matrix of Fig. 2 is introduced in order to generate the set of correlated complex-valued Gaussian variables:

$$
H_{c}\left(t_{t} ; f_{j}\right)=H_{C, t, j}=\sum_{k=1}^{\prime} a_{j, k} H_{v}\left(t_{i} ; f_{k}\right), J \in[1, N L]
$$

conditioned to

$$
m_{n, l}=E\left[H_{c, i, n}^{*} \cdot H_{C, i, l}\right]=\frac{1}{1+j 2 \pi(l-n) S_{f} D_{s}}
$$

By solving these equations we obtain the coefficients of the transformation matrix as

$$
\begin{aligned}
& a_{k, k}=\sqrt{m_{k, k}-\sum_{i=1}^{k-1}\left|a_{k, i}\right|^{2}} \\
& a_{m, k}=\frac{m_{k, m}^{*}-\sum_{i=1}^{k-1} a_{k, l}^{*} a_{m, l}}{a_{k, k}^{*}}
\end{aligned}
$$

that can be calculated iteratively beginning with the elements of the first column, $a_{i, 1}$, and continuing until the NLth column, $\mathrm{a}_{\mathrm{i}, \mathrm{NL}}$.

\section{COMPUTER SIMULATION RESULTS}

In this Section a Monte Carlo computer simulation is carried out in order to assess the performance of the system presented in Sections 2 and 3. In particular we simulate the FH-Rate $2 / 3$ Trellis Coded-8DPSK modulation scheme with the 8 state Ungerboeck code [1] [2]. We investigate the dependence of BER on signal to noise ratio, hopping-frequency separation and number of hopping-frequencies available in the system. In all the cases we consider non-frequency selective mobile radio channels, a $L=20$ symbol path memory in the Viterbi decoder, a maximum doppler shift frequency of $\mathrm{f}_{\mathrm{d}}=5 \mathrm{~Hz}$ and a bit rate of $R_{b}=64 \mathrm{Kbps}$ (it could be greater, anyway). Also, in order to emphasize only the coding and diversity effects, perfect automatic frequency control (AFC) and symbol synchronization are assumed. Typically, at least 1000 symbol enrors are associated with each data point.

Fig. 3 shows the BER-performance of the FH-Rate $2 / 3$ 8-state TCM-8DPSK system versus signal to noise ratio with normalized hopping-frequency separation, $S_{\mathrm{f}} \cdot \mathrm{Ds}$, as parameter. These results are for a number of hopping-frequencies equal to the path memory of the soft Viterbi decoder. As it can be shown, for normalized hopping-frequency separations greater than 0.15 , the degradation of the system BER-performance with respect to the null-correlation case is hardly significant. In particular, for $S_{f} \cdot D s=0.15$ and at a BER of $10^{-3}$, of interest in digital speech transmission, the coding gain degradation due to the non-null correlation effects is approximately equal to $1.5 \mathrm{~dB}$. and increases to $3 \mathrm{~dB}$ for a normalized hopping-frequency separation of 0.10 . Thus, for delay spreads ranging from $25 \mathrm{~ns}$ to around $150 \mathrm{~ns}$, typical of indoor mobile radio environments, the hopping-frequency separation necessary to obtain a coding gain degradation less than $1.5 \mathrm{~dB}$ at a BER of $10^{-3}$ should range from $6 \mathrm{MHz}$ to $1 \mathrm{MHz}$, respectively.

In Fig. 4 the effects resulting from the unavailability of sufficient bandwidth are shown. That is, by reducing the interleaver buffer size to $\mathrm{NL}<\mathrm{L}$, the allocated bandwidth is now $N L \cdot S_{f}$ instead of $L \cdot S_{f}$, where $S_{f}$ is chosen to allow practically uncorrelated responses among the different frequency channels involved. It can be seen that for $\mathrm{NL}=5$, that is, with only five hopping-frequencies, the results practically coincide with those obtained for $\mathrm{NL}=\mathrm{L}=20$. Thus, by reducing $75 \%$ of the allocated FH band the degradation of the system BER-performance is only $1 \mathrm{~dB}$ at a BER of $10^{-3}$. Even with only four hoppingfrequencies, this degradation does not exceed $2 \mathrm{~dB}$. It is worthy of point out that the case of NL=1 in Fig. 4 corresponds to a transmission system that does not use interleaving. The FH interleaving gain is then as much as $13.5 \mathrm{~dB}$, approximately, at a BER of $10^{-3}$. 


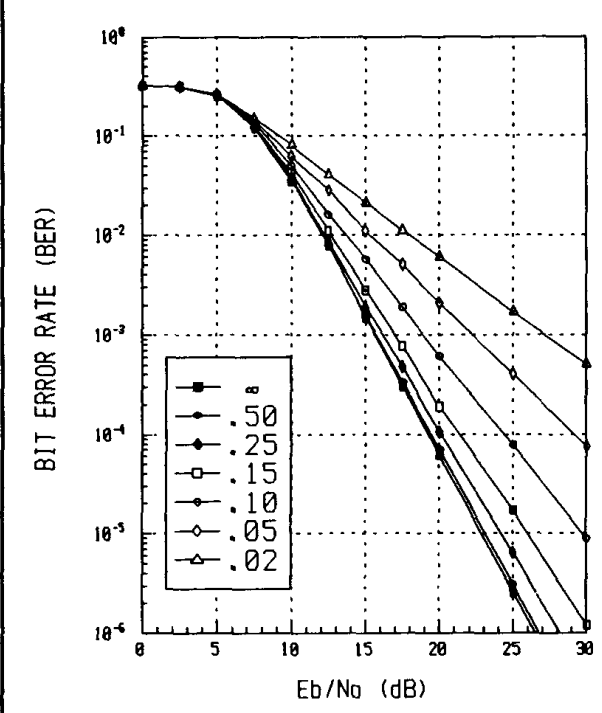

Fig 3. BER-Performance of Rate 2/3 8-State FH-TCM-80PSK systen with normalized hopping-frequency separation (Sf Ds) as par ameter. (Rb=64kbit/s, $L=20, N=20, i d=5 H_{2}$ )

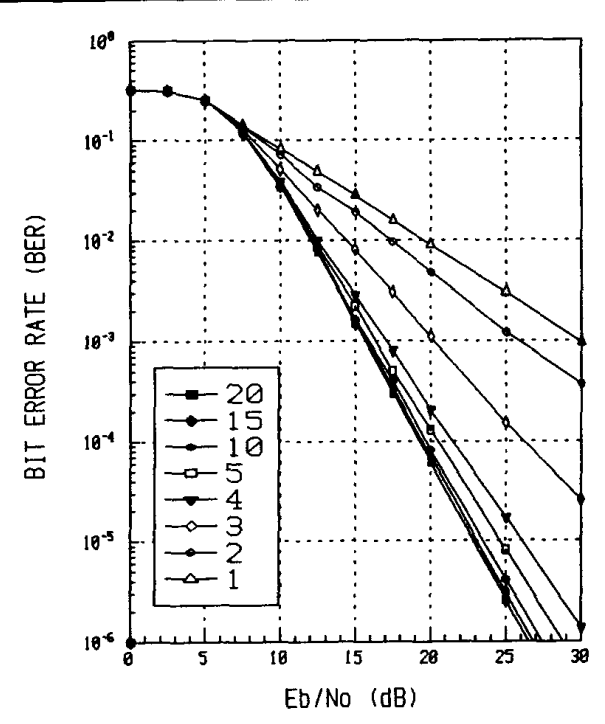

Fin 4. BER-Per formance of Rate 2/3 8-State FH-TCH-8DPSR with the number of frequency channels, assumed to be uncarrelated, as par ameter. (Rb $=64$ Kbit $/ 5, L=20, \mathrm{fd}=5 \mathrm{Hiz}$ )

\section{CONCLUSIONS}

In this paper we have analized the performance of a TDMA Slow FH-Rate 2/3 8-state TCM-8DPSK system on the GWSSUS channel. BER has been obtained, through Monte Carlo computer simulation, as a function of SNR and with normalized frequency-hopping separation and number of hopping-frequency channels as parameters. We have found that the degradation of the system BER-performance with respect to the null-correlation case is, for normalized hopping-frequency separations greater than 0.15 , hardly significant. Furthermore, by reducing $75 \%$ of the allocated $\mathrm{FH}$ band the degradation of the system BER-performance is only $1 \mathrm{~dB}$ at BER of $10^{-3}$, of interest in digital speech transmission. We have also pointed out that the FH interleaving gain is greater than $13 \mathrm{~dB}$ at a BER of $10^{-3}$.

\section{AGREEMENTS}

This work was supported by CICYT (Spain) under Grant TIC 880543 .

\section{REFERENCES}

[1] G. UNGERBOECK: Channel Coding with Multilevel/Phase Signals, IEEE Trans. on Inf. Theory, vol. IT-28, January 1982.

[2] G. UNGERBOECK: Trellis-Coded Modulation with Redundant Signal Sets. Part I: Introduction. Part II: State of the Art. IEEE Communications Magazine, vol. 25, No. 2, February 1987.

[3] G. FEMENIAS and R. AGUSTI: Switched-Diversity Trellis-Coded 8-DPSK for mobile radio applications, Proceedings IEEE VTC'90, Orlando, Florida.

[4] F. EDBAUER: Performance of Interleaved Trellis-Coded Differential 8-PSK Modulation over Fading Channels, IEEE Journal on Sel. Areas in Comm., vol 7, no. 9, December 1989.

[5] W.C. JAKES, ed., Microwave Mobile Communications, New York: John Wiley and Sons, 1974. 